\title{
Estimadores de fase óptica en sistemas modernos de comunicaciones Homodinas
}

\section{Optical Phase Estimators in Modern Homodyne Communications Systems}

\author{
A. Arvizu-Mondragón \\ CICESE. Ensenada, Baja California. México. \\ E-mail:arvizu@cicese.mx \\ F.J. Mendieta-Jiménez \\ CICESE. Ensenada, Baja California. México. \\ E-mail:mendieta@cicese.mx \\ J. de D. Sánchez-López \\ Facultad de Ingeniería de la Universidad Autónoma de Baja California (UABC). \\ E-mail:sanchezl@cicese.mx
}

(Recibido: agosto de 2006; aceptado: agosto de 2008)

\section{Resumen}

En este trabajo reportamos la síntesis de un estimador óptimo de la fase de una portadora con modulación de fase aleatoria detectada coherentemente. Nuestra aplicación está orientada a la detección homodina con modulación binaria de la fase en sistemas modernos de comunicaciones ópticas. Mecanizamos un estimador que opera sobre un observable en banda base con ruido aditivo, usando un modelo en variables de estado para el canal de comunicaciones y técnicas de estimación óptimas. Evaluamos con simulaciones el desempeño de la estructura general y versiones simplificadas del estimador a través del valor cuadrático medio de su error de fase. De estos resultados se obtuvieron estimadores prácticos que implementamos en circuitería analógica y usando un procesador digital de señales. El estimador de "varianza constante" presentó el mejor desempeño experimental evaluado a través del valor cuadrático medio de su error de fase.

Descriptores: Estimación óptima, detección homodina, procesos de Wiener, modulación de fase, variables de estado.

\begin{abstract}
This work consists of the synthesis of an optimal estimator for a random phase modulation onto a carrier to be received by a coherent detector. Our application is oriented to homodyne detection with PSK format, in modern optical communication systems. We mechanize an estimator operating with a baseband observable in additive noise employing a state variable model for the communication channel and optimal estimation techniques. To assess the performance of the general structure and simplified versions of the estimator we made computer simulations and calculated the r.m.s. value of the phase error. With these results we get practical estimators that we implemented using analog electronic circuitry and a DSP. The "constant variance" estimator exhibited the minimum r.m.s. value of its phase error obtained experimentally.
\end{abstract}

Keywords: Optimal estimation, homodyne detection, Wiener processes, phase modulation, state variable. 
DOI: http://dx.doi.org/10.22201/fi.25940732e.2009.10n3.017

Estimadores de fase óptica en sistemas modernos de comunicaciones

\section{Introducción}

La mayoría de sistemas de comunicaciones ópticas actualmente en operación son del tipo modulación de intensidad y detección directa (muy atractivos por su relativa sencillez de implementación). Por otro lado, existen también los sistemas con detección óptica coherente con ventajas (potenciales) importantes sobre los primeros, tales como una mayor selectividad en longitud de onda, mayor sensitividad en la etapa de recepción (Ho, 2005), lo que permite (teóricamente) lograr distancias mayores en los enlaces ópticos con posibilidad de una multicanalización por división de longitud de onda (WDM) más densa (Kahn, 2006), además de una mayor eficiencia espectral (Lyubomirsky, 2006). Durante los años 80's y mediados de los 90's del siglo pasado existió una gran actividad de investigación y desarrollo tecnológico en comunicaciones ópticas coherentes (Xu et al., 2004), el cual fue disminuyendo gradualmente, debido principalmente a la aparición de los amplificadores ópticos y a la gran limitante tecnológica impuesta por el ruido de fase de las fuentes ópticas (Kazovsky et al., 1996). Sin embargo, recientemente ha resurgido el interés en dichos sistemas (Lyubomirsky, 2006, Kazovsky, 2006, Kahn, 2006), en una búsqueda por incrementar la capacidad de los sistemas WDM y en vista de nuevos desarrollos tecnológicos, tanto en el área de las fuentes ópticas, foto-receptores balanceados (Discovery Semiconductors, 2005), sistemas de procesamiento digital de señales de alta velocidad (Taylor, 2004) y aplicando técnicas novedosas de codificación y sincronización (Fibers.org, 2006).

La tendencia actual en comunicaciones ópticas coherentes se orienta principalmente a la compensación y procesamiento electrónico de las perturbaciones de la fase óptica en sistemas con modulación multifásica y diferencial (Tsukamoto et al., 2005, Taylor, 2004). En este contexto, se realiza el presente trabajo, enfocándonos en la obtención de un estimador óptimo de la fase de una portadora óptica en sistemas con detección homodina, mediante la técnica de la máxima verosimilitud y la representación en variables de estado (Snyder, 1969). Esta técnica ya ha sido empleada con anterioridad para sistemas heterodinos; sin embargo, decidimos emplearla para sistemas homodinos por tener ventajas asociadas, al costo de una mayor complejidad, pero conducente a la obtención de estimadores implementables físicamente. En particular, obtenemos el estimador de fase en sistemas con modulación binaria; un paso preliminar en el estudio de los sistemas modernos de comunicaciones ópticas multifásicos (Borne et al., 2005).

\section{Estimación de fase en sistemas modernos de comunicaciones ópticas}

La estimación de la fase de una señal inmersa en ruido tiene gran importancia en sistemas modernos de comunicaciones (Lyubomirsky, 2006, Kazovsky et al., 2006, Kahn, 2006, Camatel et al., 2004, Taylor, 2004). La estimación óptima de una fase desconocida pero no aleatoria puede realizarse maximizando una función de verosimilitud adecuada (ver por ejemplo Gittlin et al., 1992), resultando en estructuras bien conocidas tales como el lazo de amarre de fase (PLL) para portadora piloto o el lazo de Costas para portadora suprimida (Meyers et al., 1980). Desafortunadamente, los parámetros encontrados en algunos campos de detección y teoría de comunicaciones son inherentemente aleatorios, por ejemplo, los sistemas de comunicaciones ópticas coherentes donde el proceso observable puede ser representado como la señal de fotocorriente postdetección en ruido aditivo y afectada por fluctuaciones de fase aleatorias provocadas por los ruidos de fase tanto del oscilador local como el de la señal recibida (Gallion et al., 1980). Esto hace necesario el empleo de técnicas de estimación de parámetros estocásticos para la síntesis de estimadores óptimos para procesos aleatorios. La formulación en variables de estado del modelo del canal permite el tratamiento de una amplia variedad de estadísticas de señales y ruidos con modulaciones no lineales y ha sido ampliamente utilizada para canales de comunicaciones digitales y analógicos, en conjunto con principios de estimación óptima que conducen a la obtención de estimadores que presentan un buen desempeño tanto en estado transitorio como en estado estable (Snyder, 1969, Baggeroer, 1970).

Estos estimadores son obtenidos mediante la mecanización de las denominadas ecuaciones del estimador y de la varianza resultantes de la maximización de una función de verosimilutd apropiada. Han sido reportados algunos trabajos empleando este enfoque para la detección coherente de un proceso aleatorio que modula una portadora inmersa en ruido, obteniéndose estructuras implementables de estimadores para el caso donde la observación es realizada a frecuencia intermedia (detección heterodina) (Georghiades et al., 1985, Georghiades, 1986). Esto permite el filtraje de términos indeseables de alto orden que aparecen en las ecuaciones del estimador y la varianza, debidos a la naturaleza no lineal de la función de verosimilitud. En el presente trabajo aplicamos las técnicas mencionadas arriba para el caso más complejo de procesamiento en banda base (detección homodina), que es más atractivo tanto desde el punto 
de vista de economía de potencia, ancho de banda y eficiencia espectral, así como de una demodulación más simple, especialmente para los casos de formatos de modulación por corrimiento de fase (PSK).

En este caso, tenemos que tratar con una configuración de estimador más complicada en comparación con los esquemas heterodinos debido al hecho de que aparecen términos de alto orden en la función de verosimilitud que deben ser tomados en cuenta por estar en la banda de paso (banda base). Con esta finalidad, planteamos en primer lugar el modelo del canal con detección coherente en una formulación de variables de estado, y a continuación desarrollamos el análisis, obteniendo ecuaciones tanto para el estimador como para la varianza que nos permiten mecanizar un estimador óptimo.

Con el fin de comparar el desempeño del estimador sintetizado realizamos simulaciones en computadora tanto del estimador general como de versiones simplificadas del mismo. Finalmente, implementamos experimentos demostrativos de baja velocidad, tanto en circuitería analógica como mediante el empleo de un procesador digital de señales de uso general.

\section{Modelo del canal con observable en banda base y ruido de fase tipo Wiener}

En el receptor coherente de la figura 1 se recibe la señal $A_{S}(t)$ con modulación de fase y potencia promedio $P_{S}$ que es mezclada con la señal de referencia $A_{L}(t)$ (oscilador local óptico) con potencia promedio $P_{L}$, (con amplitudes $A_{S O}, A_{L O}$ y frecuencias centrales $f_{S}, f_{L}$, respectivamente) descritas por:

$$
\begin{aligned}
& A_{S}(t)=A_{S O} \operatorname{sen}\left[2 \pi f_{S} t+\varphi_{S}(t)+\theta_{S}+m_{K}(t)\right], \\
& A_{L}(t)=A_{L O} \operatorname{sen}\left[2 \pi f_{L} t+\varphi_{L}(t)+\theta_{L}\right],
\end{aligned}
$$

$\varphi_{S}(t)$ y $\varphi_{L}(t)$, son procesos independientes que modelan las fases estocásticas de las señales mezcladas; $\theta_{S}$ y $\theta_{L}$ son variables aleatorias distribuidas uniformemente en el intervalo $[-\pi, \pi]$, debidas a la ausencia de referencia de fase absoluta en transmisor y receptor. $m_{K}(t)$ : k-ésimo símbolo de la forma de onda moduladora con periodo $\mathrm{T}$. Si $f_{S}=f_{L}$, el mezclado y detección coherente (homodinaje) de $A_{S}(t)$ y $A_{L}(t)$ produce:

$$
v_{S L}(t)=I_{H} \operatorname{sen}\left[\varphi(t)+\theta+m_{k}(t)\right]
$$

con con $i_{H}=r A_{S O} A_{L O}$ : amplitud en banda base y r: responsividad del fotodetector; $\varphi(t)=\varphi_{S}(t)-\varphi_{L}(t)$ :proceso aleatorio representando las fluctuaciones de fase en el proceso de banda base y $\theta=\theta_{S}-\theta_{L}$.

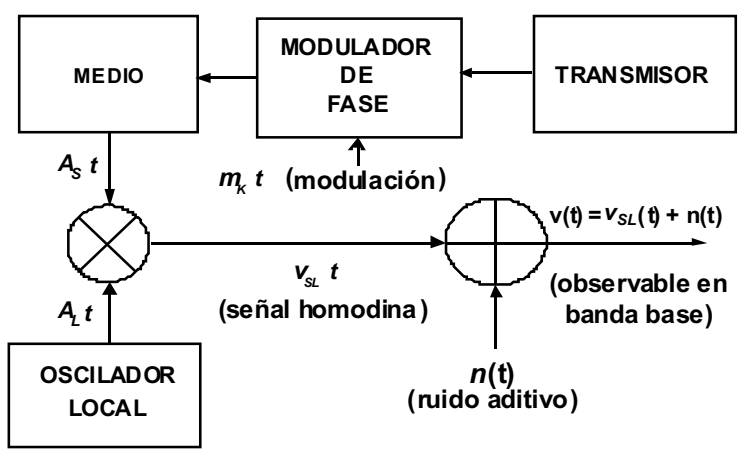

Figura 1. Modelo del canal coherente homodino

En la figura 2 se muestra el modelo en variables de estado del receptor homodino empleado para la síntesis del estimador de fase óptimo (Snyder, 1969), con el observable descrito por la siguiente ecuación de Ito (Macchi et al., 1981):

$$
v(t) d t=v_{S L}(t) d t+n(t) d t
$$

donde $n(t)=\left(K_{1}\right)^{0.5}(d \eta(t) / d t)$ es un ruido aditivo blanco Gaussiano con densidad espectral bilateral $N_{0}=q r P_{L}, \mathrm{y}$ $q$ es la carga del electrón, $\eta(t)$ es un proceso de Wiener estándar y $K_{1}=N_{0} / 2$. La fase $\varphi(t)$ puede ser escrita como:

$$
d \phi / d t=(\Delta v)^{0.5} w(t)
$$

donde $w(t)$ es un ruido blanco normalizado que genera el proceso de fase en banda base y $\Delta v=\Delta v_{S}+\Delta v_{L}$ y $\Delta v_{S}, \Delta v_{L}$, son los anchos espectrales de media potencia (FWHM) de la señal recibida y del oscilador local, respectivamente con tiempos de coherencia $t_{C S}=1 / \Delta v_{S}$ y $t_{C L}=1 / \Delta v_{L}$. Las ecuaciones (4) y (5) constituyen el modelo en variables de estado para nuestro problema de estimación; siguiendo a Snyder (1969), esta formulación nos permite aplicar las siguientes ecuaciones del estimador (6) y la varianza (7):

$$
d \hat{\phi}(t)=K_{2} E\left[e(t) v_{S L}(t)\right] v_{1}(t)
$$

donde: $K_{2}=2 / N_{0}, e(t)=\phi(t)-\hat{\phi}(t), v_{1}(t)=v(t)-E_{1}, \mathrm{Y}$

$$
E_{1}=E\left[v_{S L}(t) d t\right]
$$


$d \sum(t)=\frac{d t}{t_{c}}+K_{2} E\left[e^{2}(t) v_{2}(t)\right] v_{3}(t)-K_{2} E^{2}\left[e(t) v_{S L}(t)\right] d t$

donde:

$$
\begin{aligned}
& v_{2}(t)=v_{S L}(t)-E\left[v_{S L}(t)\right], \mathrm{y} v_{3}(t)=v(t) d t-E_{1} . \\
& \hat{\phi}(t)=E\left[\phi(t) / v(t), m_{j}\right]
\end{aligned}
$$

es el estimado del mínimo error cuadrático medio (MMSE) de $\phi(t)$ dado el proceso de observación $v(t)$, suponiendo que fue enviado $m_{j}$.

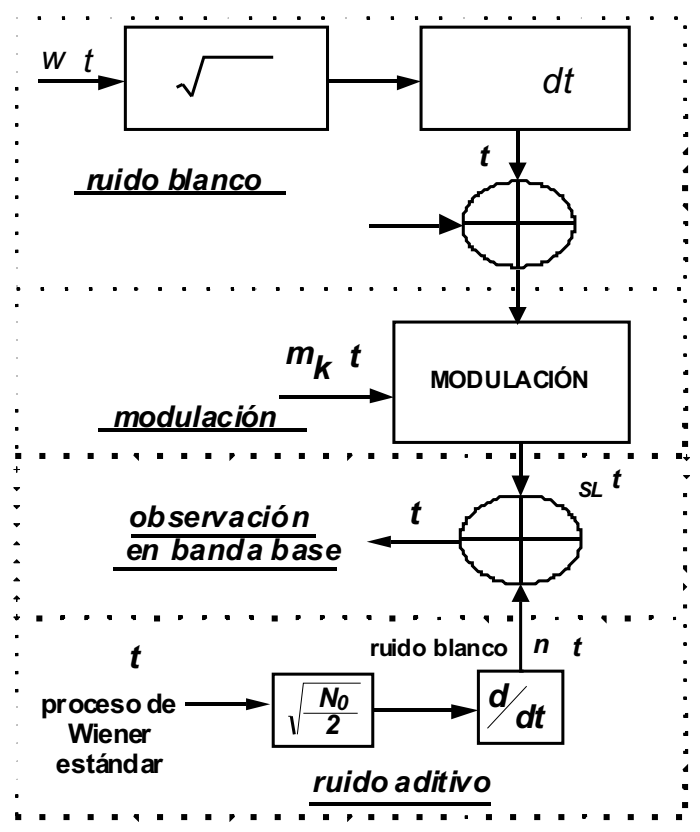

Figura 2. Modelo en variables de estado

La esperanza matemática es con respecto a la densidad condicional de $\phi(t)$ dada la observación y suponiendo que fue enviado $m_{i}: p\left[\phi(t) / v(t), m_{i}\right] e(t)=\phi(t)-\hat{\phi}(t)$ es el error de estimación, $\sum(t)=E\left[(\phi(t)-\hat{\phi}(t))^{2}\right]$ es la varianza del error de fase y el estimado de la señal es:

$$
\hat{v}_{S L}(t)=E\left[v_{S L}(\phi(t), t, m) / v(t), m_{j}\right], j=0,1 .
$$

La evolución temporal de la densidad condicional del proceso de fase $\phi(t)$ descrita por una ecuación de Fokker-Planck (Meyr et al., 1990, Georghiades, 1985) no es resoluble o implementable, por lo tanto desarrollamos las ecuaciones del estimador y la varianza usando funciones características del error de fase y obtenemos versiones simplificadas para dichas ecuaciones con un error de fase con distribución Gaussiana, empleando la notación $\phi^{*}$ y $\Sigma^{*}$ para dichos estimados:

$$
\begin{aligned}
& \phi^{*}(t)=\int k_{3} \sum^{*}(t) v(t) e_{2}^{*}(t) \cos \left(\phi_{1}^{*}(t)\right) d t- \\
& -\int k_{4} \sum^{*}(t) e_{1}^{*}(t) \operatorname{sen}\left(2 \phi_{1}^{*}(t)\right) d t \\
& \sum^{*}(t)=\int \frac{d t}{t_{c}}-\int k_{3} \sum^{* 2}(t) e_{2}^{*}(t) \operatorname{sen}\left(\phi_{1}^{*}(t)\right) v(t) d t- \\
& \int 2 K_{4} \sum^{{ }^{*}}(t) e_{1}^{*}(t) \cos \left(2 \phi_{1}^{*}(t)\right) d t
\end{aligned}
$$

donde:

$$
\begin{aligned}
& k_{3}=4 I_{H} / N_{0}, e_{1}^{*}(t)=\exp \left(-\sum^{*}(t)\right), e_{2}^{*}(t)= \\
& \exp \left(-\sum^{*}(t) / 2\right), k_{4} I_{H}^{2} / N_{0}, \mathrm{y} \phi_{1}^{*}(t)=\phi^{*}(t)+m_{j}
\end{aligned}
$$

En estas ecuaciones ("acopladas") del estimador y la varianza, respectivamente, observamos la presencia de términos del doble de la fase, no presentes en los estimadores heterodinos convencionales. Nótese que la simplificación de las ecuaciones (9) y (10) con $\sum^{*}(t)=\sum^{*}=$ constante da un estimador que denominaremos de "varianza constante" en el cual el valor de $\sum^{*}$ requiere ser calculado solamente una vez y es almacenado para ser empleado en los cálculos pertinentes. Esta estructura es similar a la obtenida para un sistema con subportadora con modulación PSK (Clark, 1972) que incluye una rama de retroalimentación con un término del doble de la fase $(\operatorname{sen}(2 \phi))$. Una simplificación mayor de la ecuación (9) ignorando términos de alto orden conducen, como era de esperarse, a la estructura de un PLL tradicional:

$$
\phi_{P L L}^{*}(t)=\int k_{3} \sum^{*} \exp \left(-\frac{\sum^{*}}{2}\right) \cos \left(\phi_{1}^{*}(t)\right) v(t) d t
$$

Desafortunadamente esta estructura que ignora los términos de mayor orden, no es aplicable al caso homodino. 
DOI: http://dx.doi.org/10.22201/fi.25940732e.2009.10n3.017

A. Arvizu-Mondragón, F.J. Mendieta-Jiménez y J. de D. Sánchez-López

\section{Evaluación del desempeño}

En un sistema heterodino, el error de fase puede plantearse mediante una evolución de Fokker-Planck (Georghiades,1985), pudiendo encontrarse una forma explícita para la función densidad de probabilidad (pdf) del error de estado estable, (consistente en una distribución del tipo Tikhonov) así como del error de estado estable. Sin embargo, en nuestro análisis, la presencia de términos de alto orden no permite la obtención de una forma explícita para la pdf, por lo tanto, para evaluar el desempeño de nuestros estimadores ("ecuaciones acopladas", "varianza constante" y PLL) nos valimos de un criterio comúnmente empleado en este tipo de esquemas, el valor cuadrático medio del error de fase. Para lo anterior, realizamos en primer lugar, la simulación de los estimadores mediante el uso de un programa comercial (SIMULINK), y a continuación, se realizó una implementación de baja frecuencia empleando un procesador digital de señales (PDS) de propósito general. La figura 3 muestra gráficas del error de fase de los 3 estimadores en función del tiempo, obtenidas mediante simulación para dos condiciones extremas de operación: a) poco ruido de fase (ancho de banda, $\mathrm{PNBW}=20$ $\mathrm{kHz}$ ) y alta relación señal a ruido (SNR) de $40 \mathrm{~dB}, \mathrm{~b}$ ) para $\mathrm{PNBW}=20 \mathrm{MHz}$ y $\mathrm{SNR}=0 \mathrm{~dB}$; observando que el estimador de "ecuaciones acopladas" tiene prácticamente el mismo desempeño que el estimador de "varianza constante" (sus gráficas están superpuestas) para ambas condiciones, teniendo ambos mejor desempeño (el error de fase tiende a cero) que el PLL que presenta una deriva del error de fase alcanzando valores entre $0.5 \mathrm{y}$ -1.5 radianes (valor inaceptable en aplicaciones prácticas). Al realizar simulaciones para otras combinaciones de relación señal a ruido se observó un comportamiento similar, lo que nos conduce a pensar que los estimadores de "ecuaciones acopladas" y de "varianza constante" tendrán un buen desempeño para condiciones prácticas de operación. Con fines comparativos, en la figura 4 se muestra el comportamiento de los estimadores de "varianza constante" y PLL implementados en un PDS, notando como era de esperarse, un mejor desempeño del primero (el estimado $\left(\phi^{*}(t)\right)$ es muy parecido a la fase original $(\phi(t)))$.

Por otro lado, con la finalidad de evaluar la factibilidad de realización física, implementamos la estructura de "varianza constante" mediante circuitería analógica (ver figura 5.a), mostrando en la figura 5.b un oscilograma típico para ruido de fase con un ancho de línea de $0.5 \mathrm{kHz}$ en ausencia de ruido aditivo, donde se observa que el estimado $\left(\phi^{*}(t)\right)$ sigue fielmente la mayoría de las variaciones de la señal de ruido de fase $(\phi(t))$.

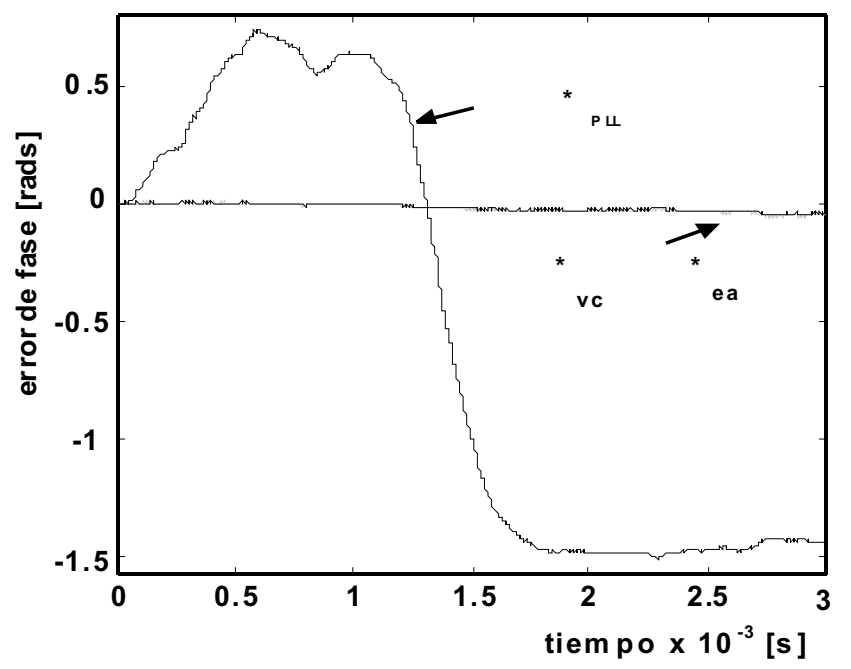

(a)

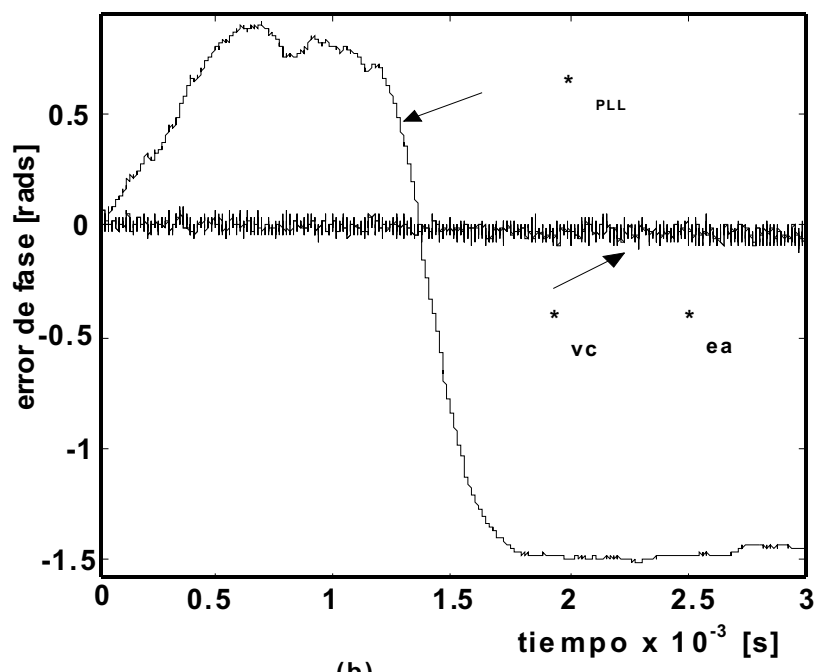

(b)

Figura 3. Gráficas del error de fase para los tres estimadores (ea: ecuaciones acopladas; vc: varianza constante y $P L L$ ) para dos condiciones de operación diferentes 


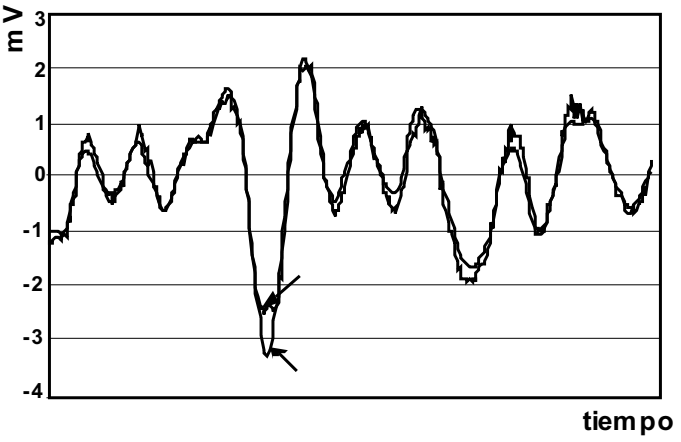

(a)

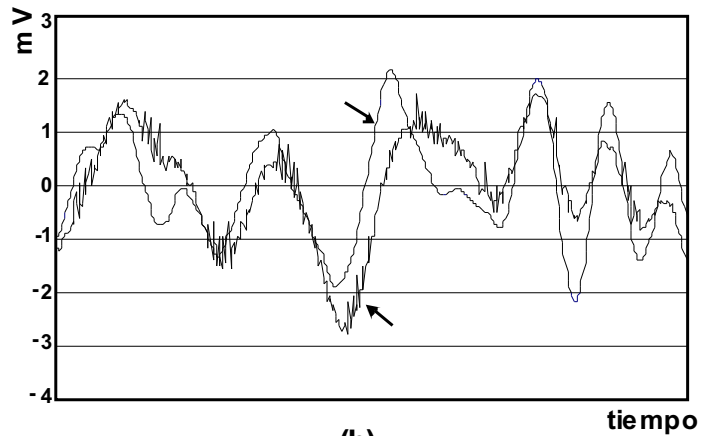

(b)

Figura 4. Formas de onda obtenidas para la implementación en un PDS para los estimadores tipo: a) varianza constante y b) PLL.

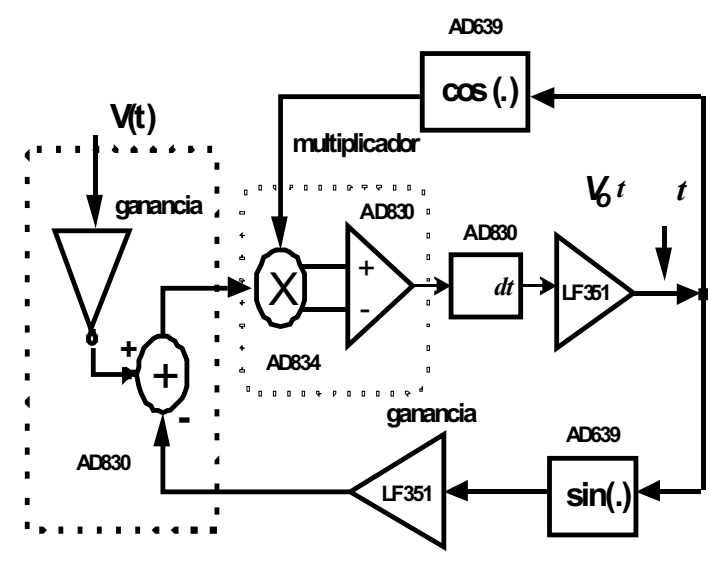

(a)

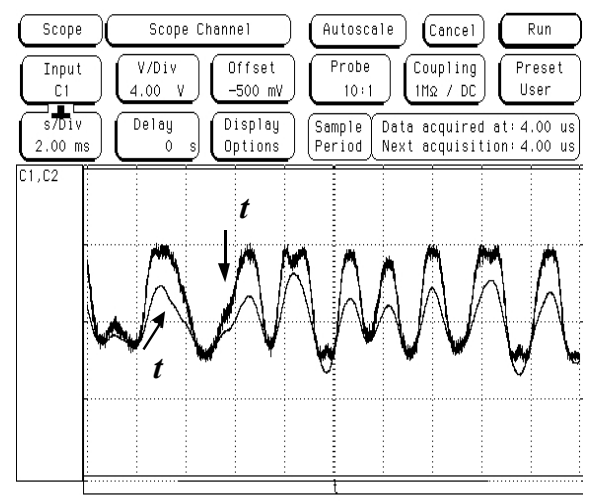

(b)

Figura 5. Estimador de varianza constante: a) diagrama a bloques del circuito eléctrico, b) oscilograma.

\section{Conclusiones}

En este trabajo empleamos la formulación en variables de estado del canal coherente y técnicas de estimación óptima para obtener una estructura estimadora para un proceso de ruido de fase modulante en la presencia de ruido aditivo. El estimador de fase se obtuvo para sistemas con modulación bifásica, como un paso preliminar en el estudio de sistemas multifásicos y diferenciales que es la tendencia en sistemas modernos de comunicaciones ópticas. Si bien se han reportado varias estructuras para la estimación de un proceso aleatorio modulando a una portadora inmersa en ruido, todas estas estructuras requieren procesamiento a una frecuencia intermedia (detección heterodina); la principal contribución de nuestro trabajo es la síntesis y mecanización de un estimador que puede ser obtenido a partir de un observable en banda base, requerido en la detección homodina. Nuestras estructuras simplificadas conducen a estimadores prácticos físicamente realizables en circuitería analógica y en PDS. El procesamiento óptimo en nuestros estimadores no intenta remover la modulación tal como ocurre en el PLL convencional y en receptores tipo lazo de Costas o lazo cuadrador, sino más bien produce un estimado del proceso de señal para cada una de las posibles modulaciones y usa entonces estos estimados en la mejor forma para identificar cuál es la información enviada más probable. Dado que los términos de alto orden en el funcional de verosimilitud no pueden ser eliminados, la estructura resultante es compleja; por esta razón mecanizamos versiones simplificadas tales como los estimadores de "varianza constante" y PLL y realizamos simulaciones en computadora. Aunque el experimento demostrativo fue implementado a una 
baja velocidad de bit, es de esperar que el comportamiento del estimador sea similar a más altas velocidades siempre y cuando se disponga de los elementos electrónicos analógicos adecuados y/o de un procesador digital de señales de alta frecuencia, ya que en la solución de las ecuaciones del estimador no se hizo ninguna restricción en cuanto a la velocidad de bit. Por otro lado, el siguiente paso a seguir consiste en el planteamiento en variables de estado del modelo del canal para el caso de modulación multifase y/o diferencial y la solución de las correspondientes ecuaciones del estimador y la varianza.

\section{Apéndice}

Basándonos en (Georghiades, 1985), el estimador MMSE de la señal $v_{S L}$ en la ecuación (8) es expresada en términos del estimado MMSE del error de fase como:

$$
\hat{v}_{S L}(t)=I_{H} \operatorname{Im}\left\{\exp \left(i\left(m_{j}+\hat{\phi}(t)\right)\right) E[\exp (i e(t))]\right\}
$$

Observando que $M_{e}(u)=E[\exp (i u e(t))]$ es la función característica del error de fase y después de algunas manipulaciones algebraicas llegamos a la expresión:

$$
\hat{v}_{S L}(t)=v_{S L}\left(\hat{\phi}(t), t, m_{j}\right) M_{e}(1) .
$$

Por otro lado, en la ecuación (6) del estimador, podemos expresar la esperanza matemática como:

$$
\begin{aligned}
& E\left\{\left(\left[\phi(t)-\hat{\phi}(t) v_{S L}(t)\right)\right\}=I_{H} \operatorname{Im}\left\{\exp \left(i\left(\hat{\phi}(t)+m_{j}\right)\right)\right\}\right. \\
& E[e(t) \exp (i e(t))]
\end{aligned}
$$

empleando:

$$
\begin{aligned}
& \frac{d}{d \hat{\phi}} v_{S L}\left(\hat{\phi}, t, m_{j}\right)=I_{H} \cos \left(\hat{\phi}(t)+m_{j}\right) \\
& \frac{d}{d u} M_{e}(u)=\dot{M}_{e}(u)=E[i e(t) \exp (\text { iue }(t))],
\end{aligned}
$$

la esperanza matemática (A.3) puede ser escrita como:

$$
E\left\{(\phi(t)-\hat{\phi}(t)) v_{S L}(t)\right\}=-\frac{d}{d \hat{\phi}(t)} v_{S L}\left(\hat{\phi}, t, m_{j}\right) \dot{M}_{e}(1)
$$

también necesitamos el siguiente producto para la ecuación (7) de la varianza:

$$
(\phi(t)-\hat{\phi}(t))^{2} v_{S L}(t)=e^{2}(t) I_{H} \operatorname{sen}\left(\phi(t)+m_{j}\right)=
$$

$$
I_{H} \operatorname{Im}\left\{\exp \left(i\left(\hat{\phi}(t)+m_{j}\right)\right) e^{2}(t) \exp (i e(t)\},\right.
$$

y empleando:

$$
\ddot{M}(u)=\frac{d^{2}}{d u^{2}} E[\exp (i u e(t))]=-E\left[e^{2}(t) \exp (i u e(t))\right],
$$

la esperanza matemática de la ecuación (A.7) es:

$$
\begin{aligned}
& E\left\{(\phi(t)-\hat{\phi}(t))^{2} v_{S L}(t)\right\}= \\
& I_{H} E \operatorname{Im}\left\{\exp \left(i\left(\hat{\phi}(t)+m_{j}\right)\right)\left(-\ddot{M}_{e}(1)\right)\right\}= \\
& -v_{S L}\left(\hat{\phi}(t), t, m_{j}\right) \ddot{M}_{e}(1),
\end{aligned}
$$

usando el producto:

$$
\frac{d}{d \hat{\phi}}\left[v_{S L}\left(\hat{\phi}(t), t, m_{j}\right)\right] v_{S L}\left(\hat{\phi}(t), t, m_{j}\right)=\frac{I_{H}^{2}}{2} \operatorname{sen}\left(2\left(\hat{\phi}(t)+m_{j}\right)\right)
$$

expresamos la ecuación del estimador como:

$$
\begin{aligned}
& d \hat{\phi}(t)=-\frac{2}{N_{0}} I_{H} \cos \left(\hat{\phi}(t)+m_{j}\right) \dot{M}_{e}(1) v(t) d t \\
& +\frac{I_{H}^{2}}{N_{0}} \operatorname{sen}\left(2\left(\hat{\phi}(t)+m_{j}\right)\right) \dot{M}_{e}(1) M_{e}(1) d t
\end{aligned}
$$

donde reconocemos la relación señal a ruido:

$$
S N R=I_{H}^{2} / N_{0}
$$

Para la ecuación (7) de la varianza usaremos (A.6), (A.8) y:

$$
\left[\frac{d v_{S L}\left(\hat{\phi}(t), t, m_{j}\right)}{d \hat{\phi}}\right]^{2}=\frac{I_{H}^{2}}{2}\left(1+\cos \left(2\left(\hat{\phi}(t)+m_{j}\right)\right)\right),
$$

para expresar:

$$
\begin{gathered}
d \sum(t)=\left\{k_{t}-k_{6}\left[1+\cos 2 \hat{\phi}_{1}(t)\right]+\left[k_{7} \sum(t)+k_{9}\right]\right. \\
\left.\left[k_{8}\left[1-\cos 2 \hat{\phi}_{1}(t)\right]+k_{10} v_{S L}\left(\hat{\phi}(t), t, m_{j}\right) v(t)\right]\right\} d t \\
\operatorname{con} k_{t}=1 / t_{c}
\end{gathered}
$$




$$
\begin{aligned}
& k_{5}=I_{H}^{2} / N_{0}, k_{6}=k_{5} \dot{M}_{e}^{2}(1), \hat{\phi}_{1}(t)=\hat{\phi}(t)+m_{j}, k_{7} \\
& =M_{e}(1), k_{8}=k_{5} k_{7}, k_{9}=\ddot{M}_{e}(1), k_{10}=2 / N_{0} .
\end{aligned}
$$

(A.10) y (A.12) son las ecuaciones generales del estimador y la varianza. Debido al hecho de la dificultad de implementación en su forma general, proponemos una simplificación para el caso de un error de fase con distribución Gaussiana y obtenemos versiones simplificadas para las ecuaciones del estimador y la varianza (A.10) y (A.12), con la notación: $\phi^{*}, \Sigma^{*} y v_{S L}^{*}$ para denotar los estimados aproximados. Para el proceso Gaussiano, la función característica es: $M_{e}(u)=\exp \left[-\frac{1}{2} \sum(t) u^{2}\right]$ y

$$
M_{e}(1)=\exp \left[-\frac{\sum(t)}{2}\right]
$$

Realizando las operaciones indicadas en (A.10), y después de algunas manipulaciones algebraicas obtenemos las ecuaciones del estimador (9) y de la varianza (10), respectivamente.

\section{Referencias}

Baggeroer A.B. State Variables and Communication Theory, Res. Mon. No. 61. MIT Press Mass. 1970. Pp.198.

Borne D. Van Den, Calabro S., Jansen S.L., Gottwald E., Khoe G.D., Waardt H. Differential Quadrature Phase Shift Keying with Close to Homodyne Performance Based on Multi-Symbol Phase Estimation. IEE Seminar on Optical Fibre Communication and Electronic Signal Processing, London, December, pp. 12/1-12/7, 2005.

Camatel S., Ferrero V., Gaudino R., Poggiolini P. Optical Phase-Locked Loop for Coherent Detection Optical Receiver. IEE Elec.Lett., 40(6), march, 2004.

Clark J.R. Direct Detection Optical Receivers for Angle Modulated Signals. Int. Conf. Commun., Philadelphia, June, 1972.

Discovery Semiconductors. Discovery Semiconductors Introduces Optical Coherent Receiver Systems, 2005 [on line]. Available on:

http://www.chipsat.com/press/press42.php.

Fibers Org. Synchronous Optical OPSK Quadruples Fiber Capacity, 2006 [on line]. Available on: http://fibers.org/articles/news/8/7/3/1.

Gallion P., Mendieta F.J., Leconte R. Single Frequency Laser Phase Noise Limitation in Single Mode Optical Fiber Coherent Detection Systems with Correlated Fields. Journ. of the Opt. Soc. of Am., $72(9): 1167$ - 1170, September, 1982.
Georghiades C.N., Snyder D.L. A Proposed Receiver Structure for Optical Communication Systems that Employ Heterodyne Detection and a Semiconductor Laser as a Local Oscillator. IEEE Trans. on Comm., 33(4):382-384, April, 1985.

Georghiades C.N. An Asymptotically Optimal Receiver for Heterodyne Optical Communication. IEEE Trans. on Comm., COM-34(6):617-619, June, 1986.

Georghiades C.N. A Proposed Receiver Structure for Heterodyne Optical Communication Systems. (PhD Thesis). St. Louis Missouri, Sever Institute of Washington University, 1985. 149 p.

Gitlin R.D., Hayes J.F., Weinstein S.B. Data Communications Principles. N.Y. Plenum Press. 1992. Pp. 733.

Ho K. Phase-Modulated Optical Communication Systems. USA. Springer+Business Media, Inc. 2005. Pp.430.

Kahn J.M. Modulation and Detection Techniques for Optical Communication Systems. OSA Topical Meeting on Coherent Optical Technologies and Applications, Whistler, BC, June. 2006.

Kazovsky L., Benedetto S. Optical Fiber Communication Systems. USA. Artech House.Inc. 1996. Pp. 690.

Kazovsky G.L., Kalogerakis G. Modern Coherent Optical Communications, CLEO/OELS Conference, Long Beach, California, May, 2006.

Lyubomirsky I. Coherent Detection for Optical Duobinary Communication Systems. IEEE Phot. Techn. Lett., 18(7):868-870, April, 2006.

Macchi O., Scharf L.L. A Dynamic Programming Algorithm for Phase Estimation and Data Decoding on Random Phase Channels. IEEE Trans. on Info. Th.,Vol. IT-27(5):581 - 595, September, 1981.

Meyr H.,Ascheid G. Synchronization in Digital Communications. Vol. 1, 1990. John Wiley \& sons, N.Y., 1990. Pp.510.

Meyers M.H., Franks L.E. Joint Carrier Phase and Symbol Timing Recovery for PAM Systems. IEEE_Trans. on Comm., Vol. COM-28(8):1121 - 1129, August, 1980.

Snyder D.L. The State Variable Approach to Continuous Estimation. 1969. Res. Mon. No. 51. MIT Press, Mass., Pp.114.

Taylor M.G. Coherent Detection Method Using DSP for Demodulation of Signal and Subsequent Equalization of Propagation Impairments. IEEE Phot.Techn. Lett., 16(2): 674-676, February, 2004.

Tsukamoto S., Ly-Gagnon D., Katoh K., Kikuchi K. Coherent Demodulation of 40-Gbit/s Polarization-Multiplexed OPSK Signals with 16-GHz Spacing after 200-km Transmission. OFC/NFOEC 2005, Anaheim, California, March. 2005.

Xu C., Liu X., Wei X. Differential Phase-Shift Keying for High Spectral Efficiency Optical Transmissions. IEEE Journ. of Select. Top.in Quant. Elect., 10(2):281-293, March/April, 2004. 
DOI: http://dx.doi.org/10.22201/fi.25940732e.2009.10n3.017

A. Arvizu-Mondragón, F.J. Mendieta-Jiménez y J. de D. Sánchez-López

\section{Semblanza de los autores}

Arturo Arvizu-Mondragón. Obtuvo el título de ingeniero mecánico electricista (1987) y el grado de maestría en ingeniería eléctrica en la Facultad de Ingeniería de la UNAM (1989). Actualmente es investigador en el Centro de Investigación Científica y de Educación Superior de Ensenada, donde obtuvo el grado de doctor en ciencias en comunicaciones (2000). Sus principales áreas de trabajo son las comunicaciones ópticas inalámbricas y por fibra óptica.

Francisco Javier Mendieta-Jiménez. Obtuvo el título de ingeniero mecánico electricista en la Facultad de Ingeniería de la UNAM en 1978 y el "Certificat d'Études Supérieures" en 1980. Asimismo, el grado de "Docteur - Ingénieur" (1983) en la École Nationale Supérieure des Télécommunications de París, Francia. Ha recibido las siguientes distinciones: premio Emilio Rosenblueth de la Academia Nacional de Ingeniería de México; el premio Ericsson de Telecomunicaciones de Industrias Ericsson, México y el premio de la Academia Mexicana de Óptica. Actualmente es investigador en el Centro de Investigación Científica y de Educación Superior de Ensenada, trabajando en criptografía cuántica y sistemas de comunicaciones ópticas inalámbricas y por fibra óptica.

Juan de Dios Sánchez-López. Ingeniero electricista egresado del Instituto Tecnológico de Cd. Madero en 1988, obtuvo el grado de maestro en ciencias en 1999 en el Centro de Investigación Científica y Educación Superior de Ensenada en el área de telecomunicaciones y electrónica. Actualmente realiza la tesis doctoral en el mismo instituto. Sus áreas de interés son las comunicaciones ópticas coherentes, las comunicaciones ópticas inalámbricas y el procesamiento de señales. 